\section{Beneficios de los ejercicios físicos intensos en mujeres posmenopáusicas con osteoporosis}

La práctica habitual de ejercicios físicos que ponen en actividad a las diferentes partes del cuerpo humano es beneficiosa en cualquier momento de la vida. En particular, los ejercicios físicos ayudan a prevenir algunas de las consecuencias negativas de la menopausia, como la pérdida ósea y el elevado riesgo de enfermedades coronarias y crónicas que afectan a las mujeres en esa etapa de la vida.

Los diversos factores de riesgo que presentan las mujeres posmenopáusicas disminuyen después de realizar ejercicios físicos con constancia, aunque los mejores resultados con relación al riesgo de padecer de osteoporosis se obtienen con programas de ejercicios diseñados especialmente para estos fines.

El Estudio de Ejercicios Físicos de Erlangen para la Prevención de la Osteoporosis (EFOPS, por sus siglas en inglés) es un programa de ejercicios multipropósitos diseñado para mujeres menopáusicas con el fin de evitar la rápida pérdida ósea y mejorar el estado físico general y la calidad de la vida de este grupo de mujeres.

El EFOPS está dirigido específicamente a mantener la masa ósea de la columna vertebral y de la región proximal del fémur, por ser estos los sitios en que ocurren con mayor frecuencia las fracturas asociadas con la osteoporosis. Para favorecer el cumplimiento del programa de ejercicios, se escogió un diseño que alternaba períodos de ejercicios de alto impacto con actividades recreativas.

En este trabajo se exponen los efectos de la práctica de ejercicios físicos intensos sobre el nivel de preparación física, la densidad mineral ósea, el dolor de espalda y la concentración de lípidos en la sangre en un grupo de mujeres posmenopáusicas.

Los criterios de inclusión fueron: tener entre 48 y 60 años de edad y entre 1 y 8 años desde la menopausia y presentar osteoporosis en la región lumbar de la columna vertebral o en la cadera (según mediciones realizadas por absorciometría de rayos $\mathrm{X}$ de energía doble). Se excluyó a mujeres con antecedentes de fracturas asociadas con la osteoporosis; con enfermedades o tratamientos que afectaran al metabolismo óseo; con enfermedades inflamatorias o cardiovasculares; con muy baja capacidad física $(<75$ vatios de carga en las pruebas de ergometría); o que hubiesen participado en competencias deportivas en las dos décadas anteriores al comienzo del estudio.
En el estudio participaron 83 mujeres; de ellas, 50 cumplieron el programa de ejercicios durante 26 meses (grupo de ejercicios) y 33 sirvieron de testigo sin entrenamiento. Todas las participantes vivían en Erlangen, Alemania, y recibieron suplemento nutricional con calcio y colecalciferol, de acuerdo con su consumo nutricional individual, a manera de garantizar un consumo diario total de $1500 \mathrm{mg}$ de calcio y de 500 UI de colecalciferol.

El programa de ejercicios consistió en cuatro sesiones semanales divididas en dos sesiones supervisadas de 60 a 70 minutos cada una y dos sesiones de entrenamiento individual no supervisado de 25 minutos que se realizaban en los hogares. El programa de entrenamiento supervisado se subdividió en secuencias de calentamiento, saltos, fuerza y flexibilidad. La secuencia de saltos comenzó después de 6 meses de entrenamiento.

Se realizaron mediciones de control 2 meses antes del comienzo del programa de ejercicios (valores iniciales) y 26 meses después de comenzado el entrenamiento (valores finales). Las mediciones realizadas fueron: datos antropométricos (talla, peso, composición corporal y relación cintura:cadera); estado físico (fuerza isométrica máxima, resistencia, consumo máximo de oxígeno, producción máxima de dióxido de carbono y ventilación); densidad mineral ósea en la región lumbar de la columna vertebral (L1-L4), en la región proximal del fémur y en el antebrazo (medida por absorciometría de rayos $\mathrm{X}$ de energía doble y tomografía cuantitativa digital); marcadores séricos de lípidos (lípidos, colesterol total, colesterol ligado a lipoproteínas de baja densidad, colesterol ligado a lipoproteínas de alta densidad, triglicéridos, glucosa, ácido úrico y apolipoproteínas A1 y B).

Las participantes respondieron a un detallado cuestionario que permitió evaluar: 1) la frecuencia y la intensidad del dolor en varias articulaciones, 2) el volumen de ejercicios que practicaban, tanto durante toda la vida como inmediatamente antes de comenzar el estudio, 3) la carga diaria de actividad física en las tareas cotidianas y 4 ) los factores de riesgo de osteoporosis. A los 26 meses se completó el cuestionario con la información relacionada con los cambios ocurridos durante el período de ejercicios, específicamente: 5) las enfermedades y los medicamentos que pudieran haber afectado al metabolismo óseo, 6) los síntomas vasomotores y 7) las actividades deportivas realizadas ajenas al programa de ejercicios de EFOPS. 
No se encontraron diferencias significativas entre las medidas antropométricas iniciales y finales. Se observó un mejoramiento significativo en los parámetros que reflejaban el estado físico de las participantes en el grupo que realizaba ejercicios, mientras que en el grupo testigo dichos paramétros permanecieron sin cambio o empeoraron. Los valores de densitometría ósea se mantuvieron estables en el grupo que realizaba ejercicios, pero en el grupo testigo se observó una disminución significativa en la región lumbar de la columna vertebral y en la zona proximal del fémur, con valores significativamente inferiores a los del grupo que realizaba ejercicios al final del estudio.

El programa de ejercicios realizado redujo significativamente las concentraciones de colesterol total en $5 \%$ y de triglicéridos en $14 \%$, mientras que en el grupo testigo estos parámetros aumentaron en $4 \%$ y $23 \%$, respectivamente. De esta manera al finalizar el programa de ejercicios, los valores de colesterol total y triglicéridos del grupo de mujeres que realizaban ejercicios fueron significativamente menores que los del grupo testigo. No se observaron diferencias en los valores de colesterol ligado a lipoproteínas de alta densidad entre ambos grupos.

Se observó una tendencia descendente de los síntomas vasomotores; sin embargo, solo se encontraron diferencias significativas entre el grupo que realizaba ejercicios y el grupo testigo en relación con el estado de ánimo y el insomnio.

La intensidad y la frecuencia del dolor entre las participantes que realizaban ejercicios disminuyeron significativamente, lo que provocó que se encontraran diferencias significativas entre los dos grupos al final del estudio. A pesar de los ejercicios intensos, no se informaron cambios con relación al estado de las principales articulaciones.

Los marcadores séricos del recambio óseo no mostraron variaciones significativas en ninguno de los dos grupos. La densidad mineral ósea disminuyó $2,3 \%$ en el grupo testigo, mientras que en las participantes del grupo que realizaba ejercicios permaneció estable. En la región lumbar de la columna vertebral, los ejercicios permitieron detener el avance de la reducción de la densidad mineral ósea trabecular y aumentó significativamente la fortaleza de la corteza, gracias al aumento del grosor del hueso o al incremento de su densidad mineral ósea. Se observó una tendencia similar en la zona proximal del fémur, aunque esta fue menos pronunciada que en la columna vertebral.

La eficiencia del sistema cardiovascular, según los valores del consumo máximo de oxígeno, aumentó significativamente en las participantes en el grupo de ejercicios $(+12,4 \%)$ y disminuyó, aunque de manera no significativa $(-2,3 \%)$, en el grupo testigo.
Este trabajo demuestra que un programa de ejercicios generales intensos dirigidos especialmente a mejorar la densidad ósea en mujeres posmenopáusicas puede evitar la pérdida ósea y mejorar significativamente la fortaleza y la resistencia, además de reducir el dolor de espalda y las concentraciones de lípidos en sangre en este grupo de mujeres. (Kemmler W, Lauber D, Weineck J, Hensen J, Kalender W, Engelke K. Benefits of 2 years of intense exercise on bone density, physical fitness, and blood lipids in early postmenopausal osteopenic women. Results of the Erlangen Fitness Osteoporosis Prevention Study (EFOPS). Arch Intern Med. 2004;164:1084-91.)

\section{El liraglutide permite controlar la glucemia sin aumento de peso en pacientes con diabetes tipo 2}

La diabetes tipo 2 es una enfermedad caracterizada por la resistencia a la insulina y la presencia de células $\beta$ deficientes, y está asociada con la hiperglucagonemia, el aumento de la producción de glucosa en el hígado y la obesidad. Los pacientes con diabetes tipo 2 segregan menos cantidad de péptido 1 similar al glucagón (PG-1) durante las comidas. Diversos estudios han demostrado que la hormona PG-1 estimula la secreción de insulina, inhibe la secreción de glucagón en dependencia de los niveles de glucosa y retrasa el vaciamiento gástrico, lo que provoca la disminución del apetito y la pérdida de peso de los pacientes. Sin embargo, la vida media del PG-1 natural es muy corta (alrededor de 1 minuto) debido a la acción de la enzima dipeptidil peptidasa I. Además, se ha demostrado que para ejercer su acción, el PG-1 debe estar presente permanentemente en el torrente sanguíneo. A pesar de estos inconvenientes, esta hormona puede servir para el tratamiento de la diabetes tipo 2 .

El liraglutide es un análogo acilado del PG-1, de acción prolongada, que actúa como antagonista de los receptores de esta hormona. Estudios realizados en animales y humanos han demostrado el efecto positivo de este fármaco en la reducción de las concentraciones de glucosa en sangre y la seguridad de su empleo. La vida media del liraglutide después de una dosis única o de múltiples dosis es de aproximadamente 12 horas, tanto en personas sanas como en pacientes con diabetes tipo 2 . El régimen terapéutico se basa en una inyección diaria.

El objetivo de este estudio fue determinar la eficacia y la seguridad del liraglutide después de 12 semanas de tratamiento en pacientes con diabetes tipo 2. Para ello se realizó un ensayo clínico multicéntrico aleatorizado con doble enmascaramiento. A los pacientes se les pidió mantener durante la realización del ensayo la dieta acostumbrada y sus 